\title{
ENHANCED CLUSTERING ALGORITHM BASED ON FUZZY LOGIC (E-CAFL) FOR WSN
}

\author{
PAWAN SINGH MEHRA* MOHAMMAD NAJMUD DOJA, AND BASHIR ALAM ${ }^{\dagger}$
}

\begin{abstract}
Since longer lifetime of the network is utmost requirement of WSN, cluster formation can serve this purpose efficiently. In clustering, a node takes charge of the cluster to coordinate and receive information from the member nodes and transfer it to sink. With imbalance of energy dissipation by the sensor node, it may lead to premature failure of the network. Therefore, a robust balanced clustering algorithm can solve this issue in which a worthy candidate will play the cluster head role. In this paper, an enhanced clustering algorithm based on fuzzy logic E-CAFL is propound which is an improvement over CAFL protocol. E-CAFL takes account of the residual energy, node density in its locality and distance from sink and feed into fuzzy inference system. A rank of each node is computed for candidature of cluster coordinator. Experiments are performed for the designed protocol to validate its performance in adverse scenarios along with LEACH and CAFL protocol. The results illustrate better performance in stability period and protracted lifetime.
\end{abstract}

Key words: Energy Efficiency, WSN, Cluster Formation, Network Lifetime, Fuzzy Logic, Cluster Head

AMS subject classifications. 68M10, 94D05

1. Introduction. In current scenario, with rapid development in wireless communication, embedded computing and diverse sensor technology, WSN is emerging very swiftly. With enormous and cheap micro sensors deployed in the area of interest, WSN collects the data from surrounding and forward the same to the sink for further processing by end-users. There are several applications of WSN e.g. defence, structural monitoring, industrial monitoring, environmental monitoring, climatic and weather monitoring, natural disaster, health care etc. $[1,2,3]$. WSN is resource restricted with regard to energy, computation and communication.This network possess restricted power supply and requires proficient and proper utilization of sensor node (SN) power for longer lifespan of the network $[4,5]$. Since SN are light weight and tiny devices with low power, their transmission range is restricted to conserve energy [6]. Longer distance transmission of data is generally accomplished by multi-hoping or intermediate nodes. In some applications, by making use of auxiliary resource like solar cells, the power source of SN can be top up [7] nonetheless it is not continuous which can hamper the functioning of the device. The key addressing issues for improving the lifetime are network topology and efficient energy consumption. Cluster based schemes effectively maintains network topology by partitioning the field and forming clusters. To drag out the lifespan, designing of clustering algorithm which is efficient in conserving energy is inevitable. Either single hop or multi-hop methods are adapted for communicating sensed information to sink/base station (BS). Evidence from experiments portray that computation is lesser energy expensive than communication [9]. According to [10], for transfer of one bit, the energy dissipated is comparable to approximately $1 \mathrm{~K}$ operations performed by SN. For conserving the energy, emphasis should be given on two constituents: number of operations performed by SN and the communication method appertain. Blending diverse efficient method may result in extendable lifetime of WSN $[11,12]$.

This paper propounds enhanced clustering algorithm using fuzzy logic (E-CAFL) for protracting the network lifespan. Most of the clustering protocols are probabilistic and chooses cluster head on the basis of maximum remnant energy and its farness from BS which is not sufficient to choose the best candidate. This algorithm is distributive in nature which uses deterministic method. E-CAFL is an improvement over CAFL [13]. CAFL considers the remnant power and closeness to BS for calculation of chance of SN while selecting the $\mathrm{CH}$ whereas when the nodes choose their $\mathrm{CH}$, it uses remnant power of $\mathrm{CH}$ and closeness to their tentative $\mathrm{CH}$. Both the parameters are crucial but the density around the tentative $\mathrm{CH}$ is also equally important as more members in cluster will lead to more energy dissipation by $\mathrm{CH}$ in transmission and reception of data. Also, CAFL is randomized protocol where the election of $\mathrm{CH}$ is dependent upon random number (RN) generated by each $\mathrm{SN}$. $\mathrm{SN}$ gets elected as $\mathrm{CH}$ if its generated $\mathrm{RN}$ is less than a calculated threshold which can sometimes lead to no $\mathrm{CH}$ selection in a round as there are chances that $\mathrm{RN}$ generated by all the $\mathrm{SN}$ are greater than threshold.

${ }^{*}$ G.L.Bajaj Institute of Technology and Management, Greater Noida, India (pawansinghmehra@gmail.com)

$\dagger$ Jamia Millia Islamia, New Delhi, India 
Overcoming the limitations of CAFL, E-CAFL considers node density along with remnant power and separation distance from BS while choosing the best candidates for $\mathrm{CH}$ role. Also member nodes intelligently select their $\mathrm{CH}$ on the basis of $\mathrm{CH}$-Chance which takes into account the rank of the $\mathrm{CH}$ and distance to that $\mathrm{CH}$ nodes during cluster formation. Along with that, E-CAFL also eradicates the randomization by selecting only the p\% best candidates for $\mathrm{CH}$ candidature. For assessing the performance of E-CAFL, simulation experiments are carried out and compared with CAFL and LEACH.

Rest of the outline of this literature is as follows: Relevant work is discussed in Section 2. Preliminary discussion is done in Section 3, E-CAFL protocol is discussed in Section 4. Performance evaluation after simulation is done in Section 5. Finally, concluding remarks with summary of the contribution is discussed in Section 6 .

2. Relevant Work. In recent past, ample number of exploration and research work have been carried out on clustering protocols in WSN. In this section, key highlights of some popular and recent clustering techniques especially fuzzy logic (FL) based are talked about. LEACH is the pioneer protocol for cluster formation of SN in WSN [14]. It is a distributive protocol that makes decisions locally for the selection of CH. It is randomized in terms of rotation of $\mathrm{CH}$ role to distribute the load evenly. This protocol also performs data compression at $\mathrm{CH}$ level so as to minimize the amount of data directed to BS. First implementation of Fuzzy based clustering approach is propound in [15] which is an improvement over LEACH. Network lifetime is efficiently increased by making use of three input constituents (node centrality, degree of node and its energy) in Fuzzy Inference System (FIS). Twenty seven fuzzy if-then rules are used and selection of $\mathrm{CH}$ is done by BS using these rules. Since this approach is centralized, therefore it is not suitable for scalable networks. Author in [16] propounds CHEF protocol in which there are two input variables for FIS: nodes remnant energy and local distance. There are nine fuzzy rules to evaluate the fuzzified inputs and calculation of nodes chance to be elected as coordinator of cluster. LEACH-FL [17] employs similar approach to [15]. It considers three descriptor (farness from BS, density around node and node power level) in computing the chance of $\mathrm{CH}$ candidature. In [18], a clustering approach with fuzzy logic (FL) is propound to protract the life time of WSN. This protocol is scalable to large WSN. EAUCF [19] propound fuzzy based distributive clustering algorithm in which remnant energy and farness from $\mathrm{BS}$ is used for election of $\mathrm{CH}$. There are nine fuzzy IF-THEN rules to select tentative $\mathrm{CH}$. Each tentative $\mathrm{CH}$ calculate the competitive radius to compete for $\mathrm{CH}$ candidature. But this algorithm doesnt contemplate energy dissipation due to huge intra communication which deteriorates the performance of the protocol. DCFP[20] protocol lessens total network energy dissipation by building the network infrastructure once at the beginning and remain same throughout the lifetime. Fuzzy c means algorithm is adopted to allocate SN to most apposite cluster. MOFCA [21] is another approach in which $\mathrm{CH}$ are chosen based on remnant energy and aloofness to BS. If a $\mathrm{CH}$ is closer to $\mathrm{BS}$ then its competitive radius is greater and it can perform more task like data collection and transmission. The author [22] proposed HEEDML-FL-o which is enhancement over HEED [23] with fuzzy logic incorporation. The inputs considered for FIS are remnant energy and node density. It improves the energy efficacy by $193.84 \%$. DUCF[24] protocol ensures load balancing by forming clusters using fuzzy logic. There are three input parameters to fuzzifier: node density, distance from BS and remaining energy of node. The two output parameters are chance and size. MCFL [25] is somewhat diverse approach to form clusters. Most eligible nodes are selected as $\mathrm{CH}$ and are trusted for few rounds to minimize the number of exchange messages in clustering algorithm. Experimental results portray better performance of this algorithm than its comparatives.

The author [26] proposed FZSEP-E which divides the area of interest into zones with heterogeneous nodes. Fuzzy logic is adapted to form clusters with parameters like density, energy and farness from BS and substantially improves the lifetime of WSN. Author in [27] propound two step fuzzy system for selecting suitable CH. It uses descriptors like vulnerability index, energy, farness from BS, density, distance among $\mathrm{CH}$ and centrality and successfully balance the network energy consumption. FBECS [28] is distributive algorithm which assigns predefined probability to SN as per their farness from BS. It uses fuzzy logic to select optimal candidates for $\mathrm{CH}$ role. Author in [8] propound two methods to improve the efficacy of LEACH. It altered the threshold for best $\mathrm{CH}$ candidate selection and modified the TDMA schedule for better transmission mechanism. MACHFLFT [29] is fuzzy based algorithm for clustering heterogeneous nodes using fixed threshold to avoid re-clustering up to some rounds. The criteria for comparison is based on remnant energy, dead nodes, first dead node, half dead node and last dead node. El Alami and Najid propound CAFL[13] to enhance CFFL protocol. In this 
protocol, for the $\mathrm{CH}$ selection, two inputs i.e. remnant energy and closeness to BS are fed to FIS in order to compute rank and for the formation of cluster, two parameters which are considered for cluster formation are residual energy of tentative $\mathrm{CH}$ and closeness to that $\mathrm{CH}$ for increasing efficiency of network.

3. Preliminaries. The objective of proposing E-CAFL is to overcome the limitations of CAFL protocol and improving its efficiency. Before we begin with the description of the E-CAFL protocol, some characteristics of network system are discussed hereafter.

3.1. Some Assumptions and Network Structure. While designing the proposed protocol, some assumptions are made in this protocol which are mentioned below:

- The deployment of SN is randomized.

- The SN are homogeneous with battery level at parity

- BS as well as SN are immobile.

- The power supply to SN is irreplaceable and non-rechargeable whereas BS has continuous power supply.

- The separation distance is computed by RSSI.

- BS is kept aloof from field.

- The communication between any two devices is symmetric.

- SN will be presumed dead only if its power supply gets exhausted.

The target field is presumed to be of $100 \times 100$ size as shown in Figure 3.1. SN are scattered indiscriminately over the area of interest and the BS is located at $(175,50)$.

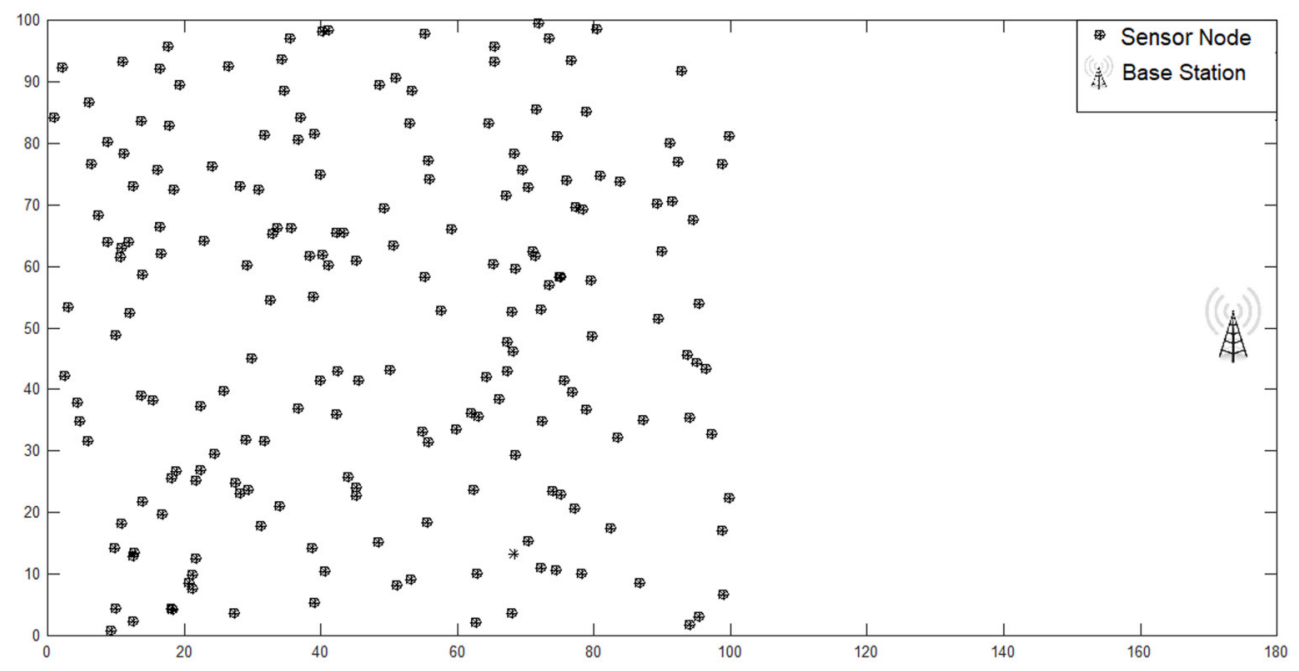

FIG. 3.1. Network Layout

3.2. Radio Energy Model. In E-CAFL, first order radio energy model is adapted which is identical to the work presented in [13]. The amount of energy dissipated for transmission and reception of $s$ bits is represented as $E_{T x}$ and $E_{R x}$ respectively. However, the system behavior is dependent upon Equations 3.1 and 3.2.

$$
E_{T x}(s, d)= \begin{cases}s E_{\text {elec }}+s \epsilon_{f s} d^{2}, & d<d_{o} \\ s E_{\text {elec }}+s \epsilon_{m p} d^{4}, & d>d_{o}\end{cases}
$$

where $d_{o}=\sqrt{\frac{\epsilon_{f s}}{\epsilon_{m p}}}$

For receiving $s$-bits data, the energy required is calculated as follows:

$$
E_{R x}(s)=E_{R x-e l e c}(s)=s E_{\text {elec }}
$$


The total energy exhausted by a $\mathrm{CH}$ in each round is calculated by Equation 3.3

$$
E_{C H}=n s\left(E_{\text {elec }}+\epsilon_{f s} d_{B S}+E_{D A}\right)
$$

where the distance between $\mathrm{CH}$ and BS is denoted by $d_{B S}$ and $n$ is the count of member nodes. Similarly, the amount of energy dissipated by a cluster member $\left(E_{C M}\right)$ is computed by Equation 3.4 in which $d_{C H}$ is the distance to its $\mathrm{CH}$.

$$
E_{C M}=s\left(E_{\text {elec }}+\epsilon_{f s} d_{C H}\right)
$$

4. E-CAFL. This section discusses enhanced clustering algorithm using fuzzy logic (E-CAFL). It is an improvement over the CAFL protocol which is discussed hereafter. First, the BS is kept aloof from the target field in both the scenario while performing simulation experiments so as to make the application suitable for situation where human reachability is rare or impossible. Second, In addition to the parameters used in CAFL while electing a node as $\mathrm{CH}$, the overhead of the $\mathrm{CH}$ is also considered in terms of node density as third parameter because it determines the intra cluster communication cost. Third, randomisation of $\mathrm{CH}$ selection is eliminated from CAFL and decision for $\mathrm{CH}$ selection is made purely on the basis of rank rather than randomly generated number. Fourth, during the cluster formation, instead of using remnant energy of $\mathrm{CH}$, we have used rank of $\mathrm{CH}$ while making any decision to choose $\mathrm{CH}$ for member nodes because it will provide profound status of the $\mathrm{CH}$ node. The E-CAFL protocol is based on rounds. Once the SN are scattered in the area of interest, E-CAFL protocol comes into play. CH selection and Cluster formation in E-CAFL is explained hereafter.

4.1. Selection of Cluster Head. In every round, only p\% $\mathrm{CH}$ are elected from alive node. To form clusters, a packet (BS_LOC) is broadcasted by BS in the field which comprises of BS coordinates and a schedule. Each SN broadcasts a packet (INFO_MSG) in field within the transmitter range as per the schedule provided by BS. After the completion of all the broadcasts, local parameters like density, remnant energy and farness from BS are computed by SN. The CH selection algorithm is described in Algorithm 1.

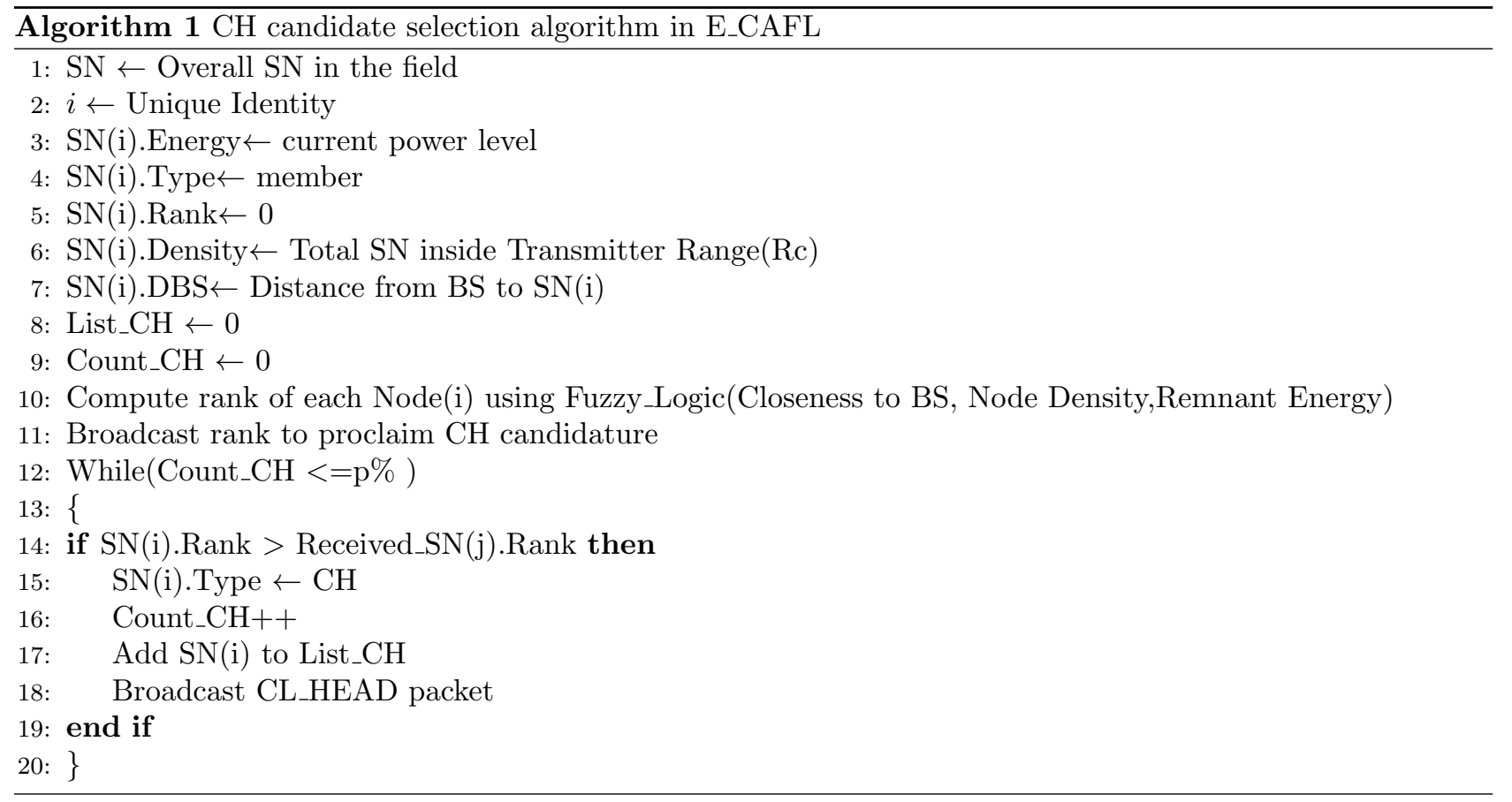

In E-CAFL, to select the $\mathrm{CH}$ candidates, fuzzy logic is incorporated. The decision making behaviour of human is efficiently handled by fuzzy logic. In order to calculate the rank of each node, three input variables; density, remnant energy and farness from BS are applied to FIS as depicted in Table 4.1. 
TABLE 4.1

Input and its linguistic variables for $\mathrm{CH}$ selection

\begin{tabular}{llll}
\hline Input Variables & \multicolumn{3}{c}{ Linguistic Variable } \\
\hline Node Density & $\operatorname{High}(\mathrm{H})$ & $\operatorname{Medium}(\mathrm{M})$ & $\operatorname{Low}(\mathrm{L})$ \\
Closeness to BS & $\operatorname{Far}(\mathrm{F})$ & $\operatorname{Medium}(\mathrm{M})$ & Near(N) \\
Remnant Energy & $\operatorname{High}(\mathrm{H})$ & $\operatorname{Medium}(\mathrm{M})$ & $\operatorname{Low}(\mathrm{L})$ \\
\hline
\end{tabular}

Triangular and Trapezoidal MF are used for intermediate and boundary variables respectively since it is simpler with faster computation. Each MF must satisfy a condition that it should range from 0 to 1 . Other MF are also there like Bell,Sigmoid, Gaussian etc. which can also be used but proposed work has shown better results with these two MF. The triangular and trapezoidal MF used are given in Equations 4.1 and 4.2 respectively.

$$
\begin{gathered}
f(y ; t, u, v)=\max \left(\min \left(\frac{y-t}{u-t}, \frac{v-y}{v-u}\right), 0\right) \\
f(y ; t, u, v, w)=\max \left(\min \left(\frac{y-t}{u-t}, 1, \frac{w-y}{w-v}\right), 0\right)
\end{gathered}
$$

These membership function (MF) of crisp input values are framed by using [13] and experimental experience as illustrated in Figure 4.1-4.3. After the fuzzification, the values obtained are provided to rule base to test for IF-THEN conditions. There are twenty seven rules as depicted in Table 4.2 which are applied to obtain the rank.

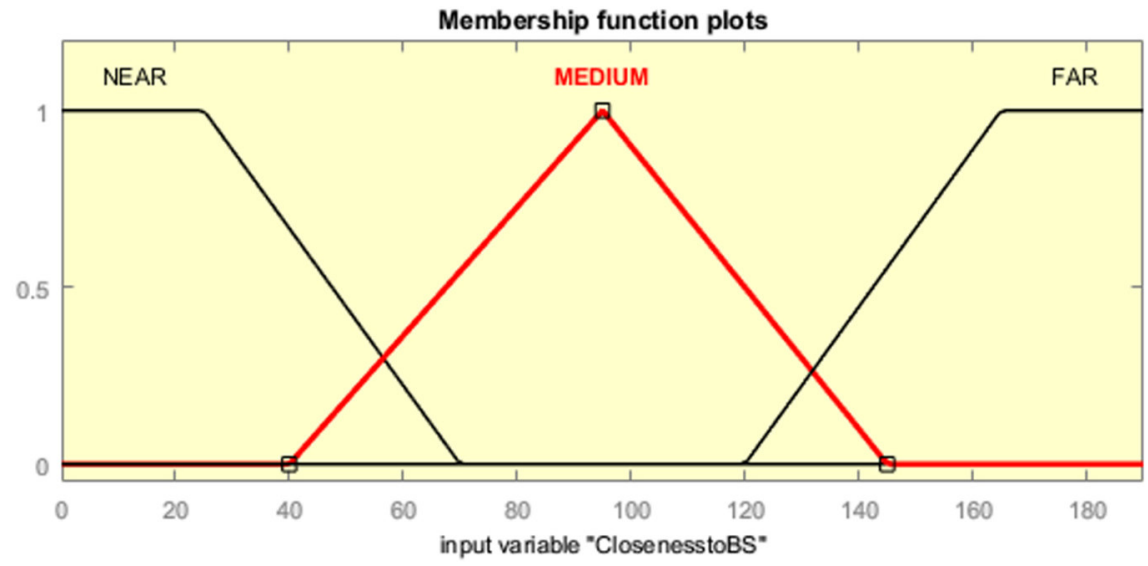

FIG. 4.1. MF-Closeness to BS

In this protocol, Mamdani inference system is adapted which is most commonly used $[22,28]$ because of its characteristics and simplicity. A value is obtained by using AND and OR operators. FIS endeavour to imitate the human inference system in making conclusion from the given set of constraints in knowledge base. The procedure of defuzzification maps the fuzzy set obtained from inference engine into crisp value for drawing conclusion. The center of area $\left(\mathrm{C}^{*}\right)$ method used for defuzzification process is given in Equation 4.3

$$
C^{*}=\frac{\int \mu_{A}(y) y d y}{\int \mu_{A}(y) d y}
$$

For crisp output, the fuzzy variables used are shown in Table 4.3. Defuzzifier changes the obtained input from inference engine into crisp set using triangular and trapezoidal MF as shown in Figure 4.4 and rank for each node is obtained. 


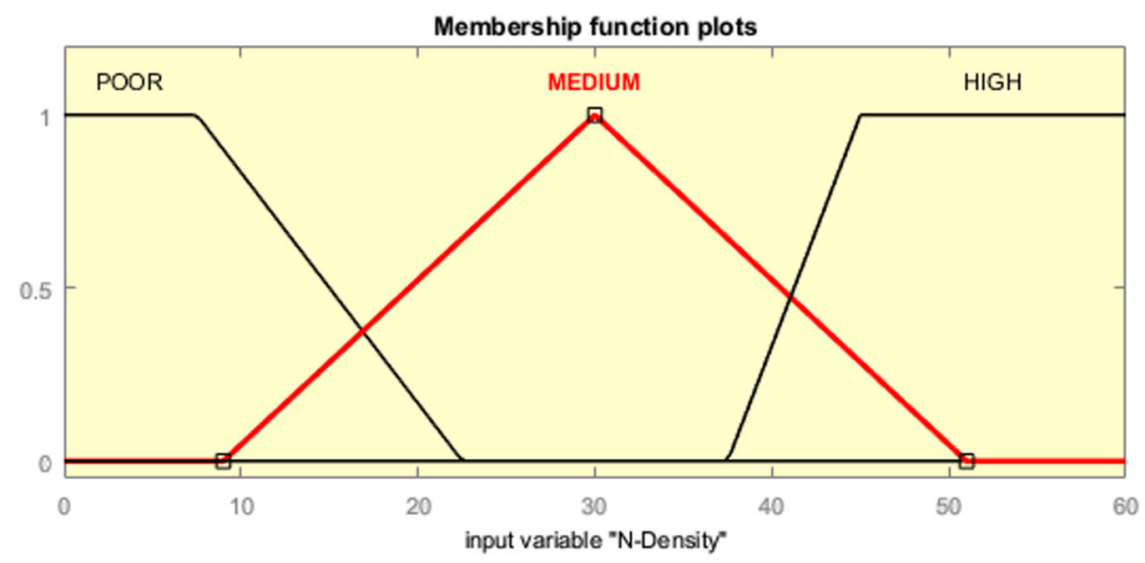

FIG. 4.2. MF- Node Density

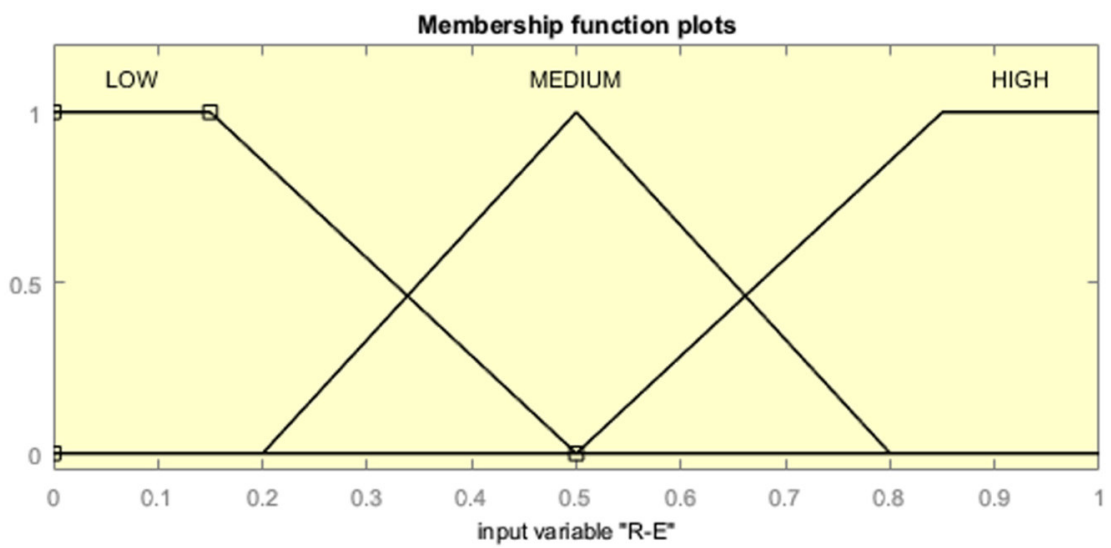

FIG. 4.3. MF-RES-Energy

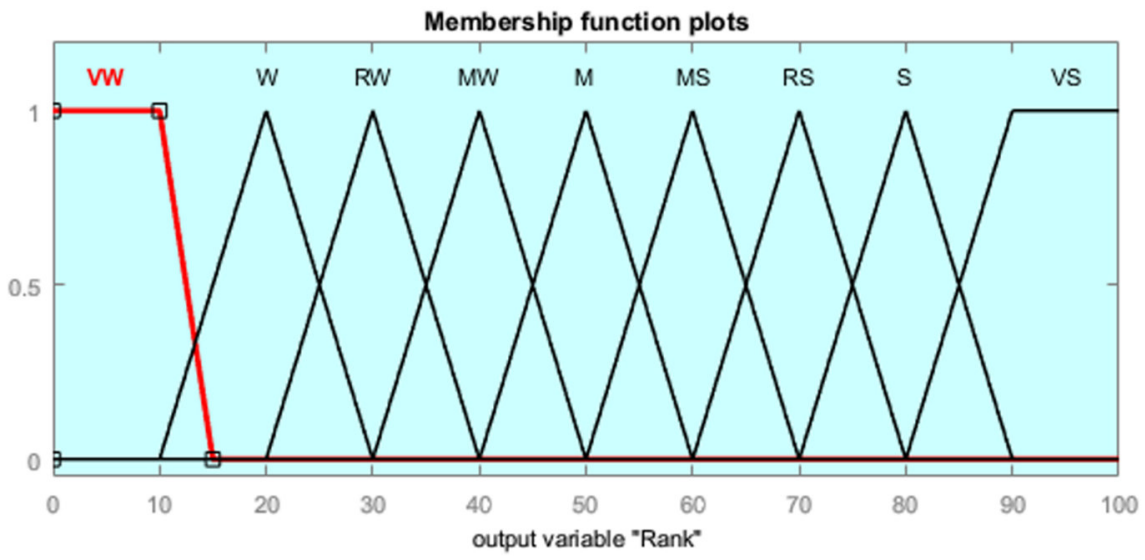

FIG. 4.4. MF-Rank 
TABLE 4.2

Fuzzy Rules for Node Ranking

\begin{tabular}{lllll}
\hline Rule No. & R_Energy & DBS & Density & Rank \\
\hline & & & & \\
1 & $\mathrm{H}$ & $\mathrm{F}$ & $\mathrm{H}$ & $\mathrm{S}$ \\
2 & $\mathrm{H}$ & $\mathrm{M}$ & $\mathrm{H}$ & $\mathrm{VS}$ \\
3 & $\mathrm{~L}$ & $\mathrm{M}$ & $\mathrm{P}$ & $\mathrm{VW}$ \\
4 & $\mathrm{H}$ & $\mathrm{N}$ & $\mathrm{H}$ & $\mathrm{VS}$ \\
5 & $\mathrm{M}$ & $\mathrm{M}$ & $\mathrm{H}$ & $\mathrm{MS}$ \\
6 & $\mathrm{H}$ & $\mathrm{F}$ & $\mathrm{M}$ & $\mathrm{RS}$ \\
7 & $\mathrm{M}$ & $\mathrm{N}$ & $\mathrm{P}$ & $\mathrm{MW}$ \\
8 & $\mathrm{~L}$ & $\mathrm{~F}$ & $\mathrm{H}$ & $\mathrm{W}$ \\
9 & $\mathrm{H}$ & $\mathrm{M}$ & $\mathrm{M}$ & $\mathrm{S}$ \\
10 & $\mathrm{M}$ & $\mathrm{M}$ & $\mathrm{M}$ & $\mathrm{M}$ \\
11 & $\mathrm{H}$ & $\mathrm{N}$ & $\mathrm{M}$ & $\mathrm{S}$ \\
12 & $\mathrm{M}$ & $\mathrm{F}$ & $\mathrm{P}$ & $\mathrm{RW}$ \\
13 & $\mathrm{~L}$ & $\mathrm{~N}$ & $\mathrm{H}$ & $\mathrm{RW}$ \\
14 & $\mathrm{~L}$ & $\mathrm{~F}$ & $\mathrm{M}$ & $\mathrm{VW}$ \\
15 & $\mathrm{H}$ & $\mathrm{F}$ & $\mathrm{P}$ & $\mathrm{MS}$ \\
16 & $\mathrm{M}$ & $\mathrm{N}$ & $\mathrm{M}$ & $\mathrm{M}$ \\
17 & $\mathrm{M}$ & $\mathrm{M}$ & $\mathrm{P}$ & $\mathrm{MW}$ \\
18 & $\mathrm{H}$ & $\mathrm{M}$ & $\mathrm{P}$ & $\mathrm{RS}$ \\
19 & $\mathrm{~L}$ & $\mathrm{~F}$ & $\mathrm{P}$ & $\mathrm{VW}$ \\
20 & $\mathrm{~L}$ & $\mathrm{~N}$ & $\mathrm{M}$ & $\mathrm{W}$ \\
21 & $\mathrm{H}$ & $\mathrm{N}$ & $\mathrm{P}$ & $\mathrm{RS}$ \\
22 & $\mathrm{M}$ & $\mathrm{F}$ & $\mathrm{H}$ & $\mathrm{M}$ \\
23 & $\mathrm{~L}$ & $\mathrm{M}$ & $\mathrm{M}$ & $\mathrm{W}$ \\
24 & $\mathrm{M}$ & $\mathrm{N}$ & $\mathrm{H}$ & $\mathrm{MS}$ \\
25 & $\mathrm{~L}$ & $\mathrm{~N}$ & $\mathrm{P}$ & $\mathrm{W}$ \\
26 & $\mathrm{M}$ & $\mathrm{F}$ & $\mathrm{M}$ & $\mathrm{MW}$ \\
27 & $\mathrm{~L}$ & $\mathrm{M}$ & $\mathrm{H}$ & $\mathrm{RW}$ \\
\hline & & & & \\
& & & &
\end{tabular}

TABLE 4.3

Output and its Linguistic Variables

\begin{tabular}{ll}
\hline Output & Linguistic Variable \\
\hline Rank & Very Strong(VS), Strong(S), Rather Strong(RS), Medium Strong(MS),Medium(M), \\
& Medium Weak(Mw), Rather Weak(RW), Weak(W),Very Weak(VW)
\end{tabular}

Once the rank of all the nodes is computed, each SN broadcasts its rank within the transmitter range as per the schedule. After the completion of all the broadcast, each sensor node compare its rank with all the received rank. If its rank is highest, it will proclaim its candidature for CH by broadcasting (CL_HEAD) packet which contains its density and remnant energy otherwise it will wait to join the optimal cluster. Thus the candidates with higher rank will be elected as $\mathrm{CH}$.

4.2. Cluster formation in E-CAFL. After the selection of $\mathrm{CH}$, rest of the nodes (Non-CH nodes) will now have to make a decision to join one of the cluster. For this, the nodes will calculate the chance of all the elected $\mathrm{CH}$ whose packet is received by the node. This cluster formation is depicted in Algorithm 2. Since, the node has CH_Rank received earlier, it will calculate its closeness to that $\mathrm{CH}$. With these two parameters, node will pass it to Fuzzy logic in order to calculate the chance of each $\mathrm{CH}$ so that it can join the optimal cluster. 
The input and its linguistic variables are listed in Table 4.4 and its MF is shown in Figure 4.5 and 4.6.
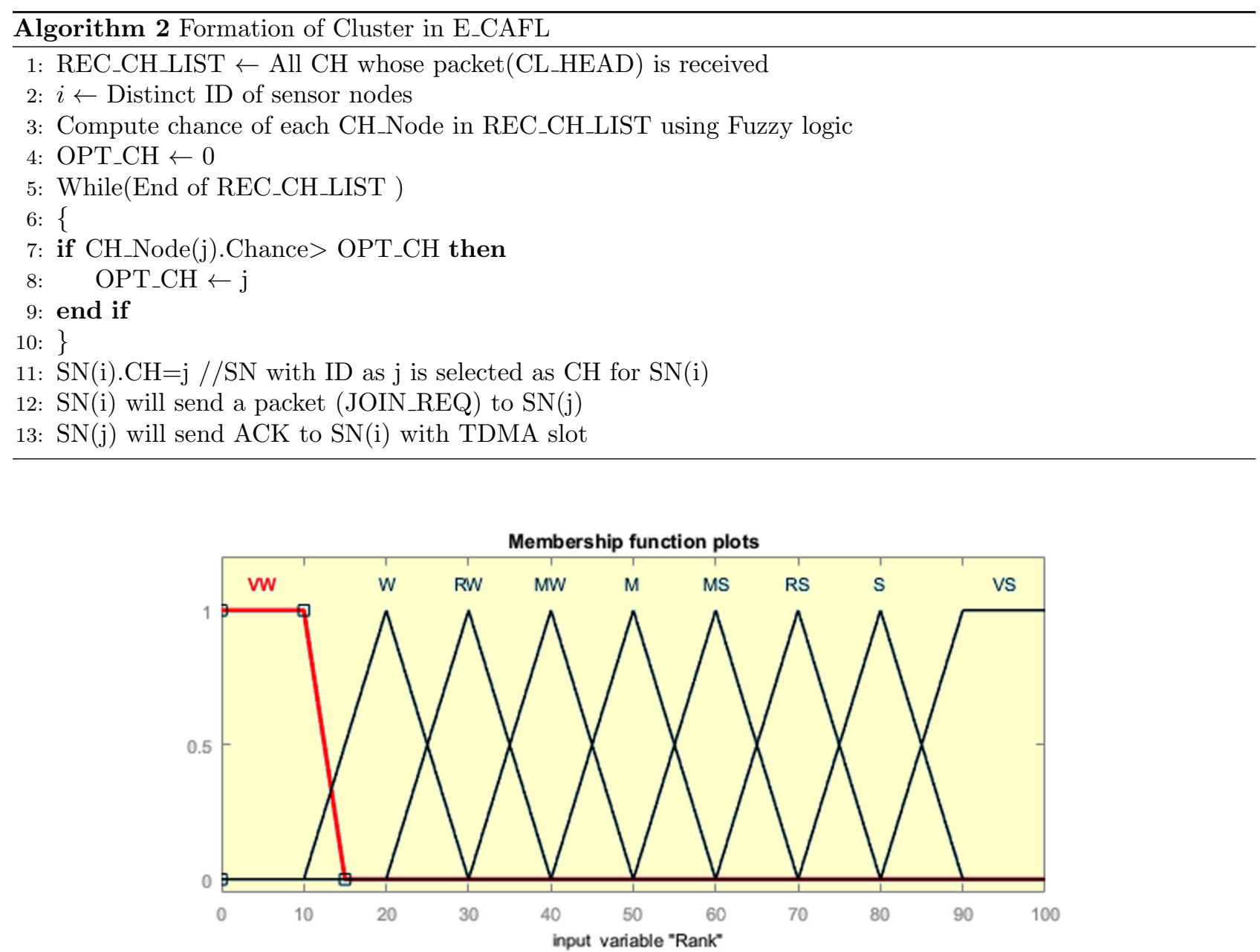

FIG. 4.5. MF-Rank-CH

TABLE 4.4

Input and its Linguistic Variables for cluster formation

\begin{tabular}{ll}
\hline Input Variables & Linguistic Variable \\
\hline CH_Rank & Very Strong(VS), Strong(S), Rather Strong(RS), Medium Strong(MS), \\
& Medium(M), Medium Weak(Mw), Rather Weak(RW), Weak(W), \\
& Very Weak(VW) \\
Closeness to CH & Far(F),Medium(M),Near(N)
\end{tabular}

After completion of fuzzification process, the values obtained are passed to rule base to test for IF-THEN rules. For calculating the chance of $\mathrm{CH}$, there are twenty seven rules to be applied as shown in Table 4.5. In this cluster formation, Mamdani model used. The defuzzification process uses $\left(\mathrm{C}^{*}\right)$ as given in Equation 4.3 and converts the received input into crisp set output. The fuzzy output variables used are similar to output linguistic variables used in Table 4.3. Triangular and Trapezoidal MF used are shown in Figure 4.7 and chance for each $\mathrm{CH}$ is obtained. 


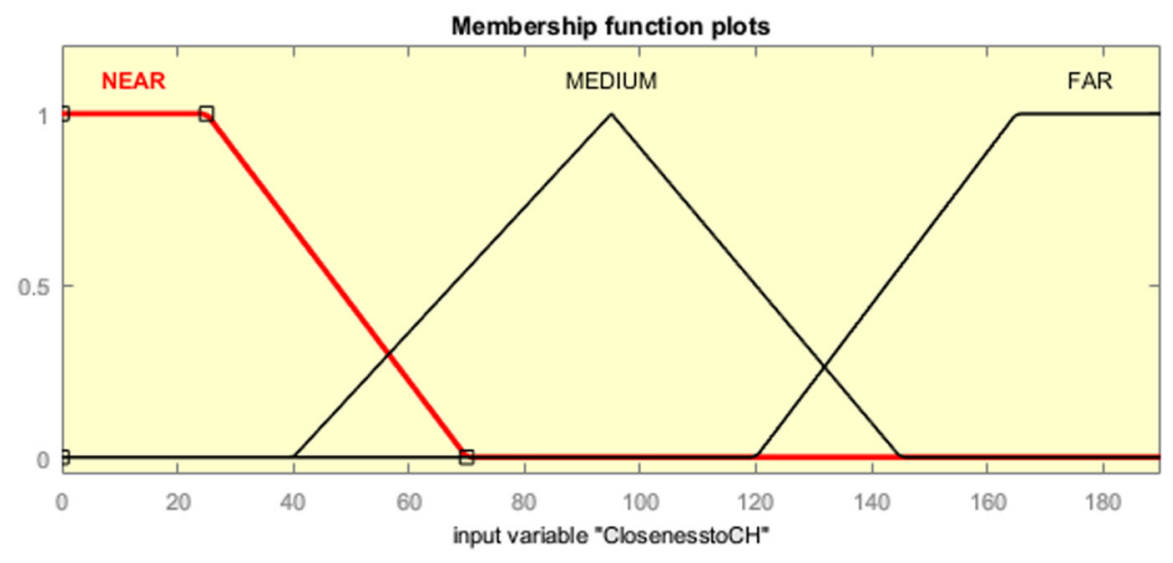

FIG. 4.6. MF-Closeness to $C H$

TABLE 4.5

Fuzzy Rules for $\mathrm{CH}_{-}$Chance

\begin{tabular}{|c|c|c|c|}
\hline Rule No. & CH_Rank & Closeness to $\mathrm{CH}$ & CH_Chance \\
\hline 1 & VS & $\mathrm{F}$ & $\mathrm{S}$ \\
\hline 2 & VS & $\mathrm{M}$ & VS \\
\hline 3 & VS & $\mathrm{N}$ & VS \\
\hline 4 & $\mathrm{~S}$ & $\mathrm{~F}$ & RS \\
\hline 5 & $\mathrm{~S}$ & $\mathrm{M}$ & S \\
\hline 6 & $\mathrm{~S}$ & $\mathrm{~N}$ & $\mathrm{~S}$ \\
\hline 7 & RS & $\mathrm{F}$ & MS \\
\hline 8 & $\mathrm{RS}$ & $\mathrm{M}$ & $\mathrm{RS}$ \\
\hline 9 & $\mathrm{RS}$ & $\mathrm{N}$ & $\mathrm{RS}$ \\
\hline 10 & MS & $\mathrm{F}$ & $\mathrm{M}$ \\
\hline 11 & MS & $\mathrm{M}$ & MS \\
\hline 12 & MS & $\mathrm{N}$ & MS \\
\hline 13 & $\mathrm{M}$ & $\mathrm{F}$ & MW \\
\hline 14 & $\mathrm{M}$ & $\mathrm{M}$ & $\mathrm{M}$ \\
\hline 15 & $\mathrm{M}$ & $\mathrm{N}$ & $\mathrm{M}$ \\
\hline 16 & MW & $\mathrm{F}$ & RW \\
\hline 17 & MW & $\mathrm{M}$ & MW \\
\hline 18 & MW & $\mathrm{N}$ & MW \\
\hline 19 & RW & $\mathrm{F}$ & W \\
\hline 20 & RW & $\mathrm{M}$ & RW \\
\hline 21 & RW & $\mathrm{N}$ & RW \\
\hline 22 & $\mathrm{~W}$ & $\mathrm{~F}$ & VW \\
\hline 23 & W & $\mathrm{M}$ & W \\
\hline 24 & $\mathrm{~W}$ & $\mathrm{~N}$ & W \\
\hline 25 & VW & $\mathrm{F}$ & VW \\
\hline 26 & VW & $\mathrm{M}$ & $\mathrm{W}$ \\
\hline 27 & VW & $\mathrm{N}$ & RW \\
\hline
\end{tabular}




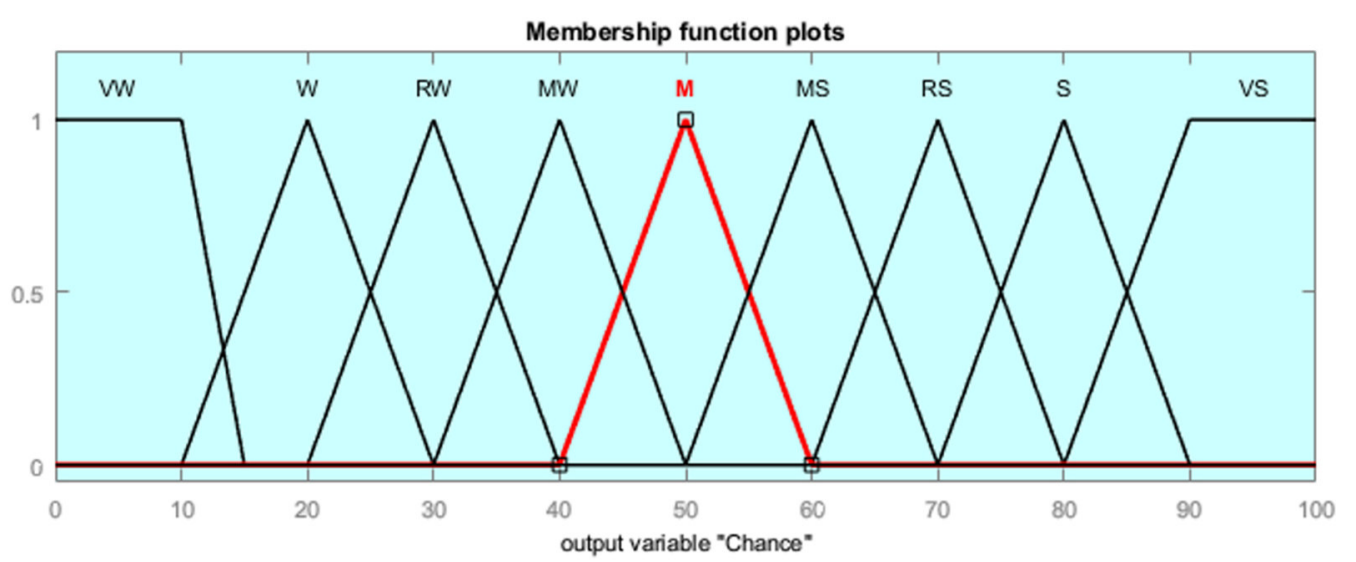

FIG. 4.7. MF-Closeness to $\mathrm{CH}$

After obtaining the chance of each $\mathrm{CH}$, node will choose to join the cluster whose $\mathrm{CH}$ is having highest chance. Node will send a (JOIN_REQ) packet to the $\mathrm{CH}$. The $\mathrm{CH}$ will accept the request and send acknowledgement packet(ACK) to the node for confirmation. In this way, all clusters are successfully formed. Afterwards, $\mathrm{CH}$ will send TDMA slot to all member nodes to collect data evading collision. After the collection of information sensed by member nodes, $\mathrm{CH}$ fuses the data for minimizing the communication cost. The fused data will transferred to BS for further processing by end user. Thus, E-CAFL will complete one round.

5. Simulation and Performance Evaluation. To analyse the performance of E-CAFL, simulation experiments are carried out and compared with LEACH and CAFL protocol. The field size is chosen to be $100 \mathrm{~m}^{2}$ with static SN scattered in random fashion. The BS is located at $(50,175)$ which is remotely located from the field in order to make the protocol widely applicable for any environment. Matrix laboratory is used for simulation work as it is easier for Fuzzy logic implementation and graphical results can be seen. The configuration of simulation parameters are shown in Table 5.1. The simulation experiments are performed extensively to obtain normalised results. For performance evaluation, two scenarios are considered in which some of the simulation parameters are varied from one another. In scenario 1 , the $\mathrm{SN}$ are available with $E_{o}=0.5 J$ and $\mathrm{N}=100$ where as in scenario $2, E_{o}=1 J$ and $\mathrm{N}=200$. For fair comparison, simulation parameters are kept similar for E-CAFL, LEACH and CAFL. The performance comparison is based on following metrics:

- Alive Nodes : It depicts the count of nodes still alive in each round.

- Average Energy : It shows the total energy of the network available in each round.

- FND, QND and HND :FND is the First Node death, QND is the Quarter Node death and HND is the Half Node Death of the protocols.

- Throughput: It is the number of information messages successfully delivered to the BS.

TABLE 5.1

Configuration of Simulation parameters

\begin{tabular}{lll}
\hline Description & Symbol & Values \\
\hline Total SN in the Field & $\mathrm{N}$ & $100 / 200$ \\
Amplifier energy for free space & $\epsilon_{f s}$ & $10 \mathrm{pJ} / \mathrm{bit} / \mathrm{m}^{2}$ \\
Amplifier energy for multipath & $\epsilon_{m p}$ & $0.0013 \mathrm{pJ} / \mathrm{bit} / \mathrm{m}^{4}$ \\
Battery level before deployment & $E_{o}$ & $0.5 \mathrm{~J} / 1.0 \mathrm{~J}$ \\
Data packet Size & $\mathrm{M}$ & $4000 \mathrm{bits}$ \\
Electronic Circuitry & $E_{\text {elec }}$ & $50 \mathrm{~nJ} / \mathrm{bit}$ \\
Data Fusion & $E_{D A}$ & $5 \mathrm{~nJ} / \mathrm{bit} / \mathrm{report}$ \\
\hline
\end{tabular}


5.1. Number of Alive Nodes. More information can be collected from the network if large count of alive SN are available in the field. As shown in Figure 5.1, a plot for number of alive nodes in the network per round is depicted for both the scenarios. The death of SN occurs at 822 round where as for CAFL and LEACH it is
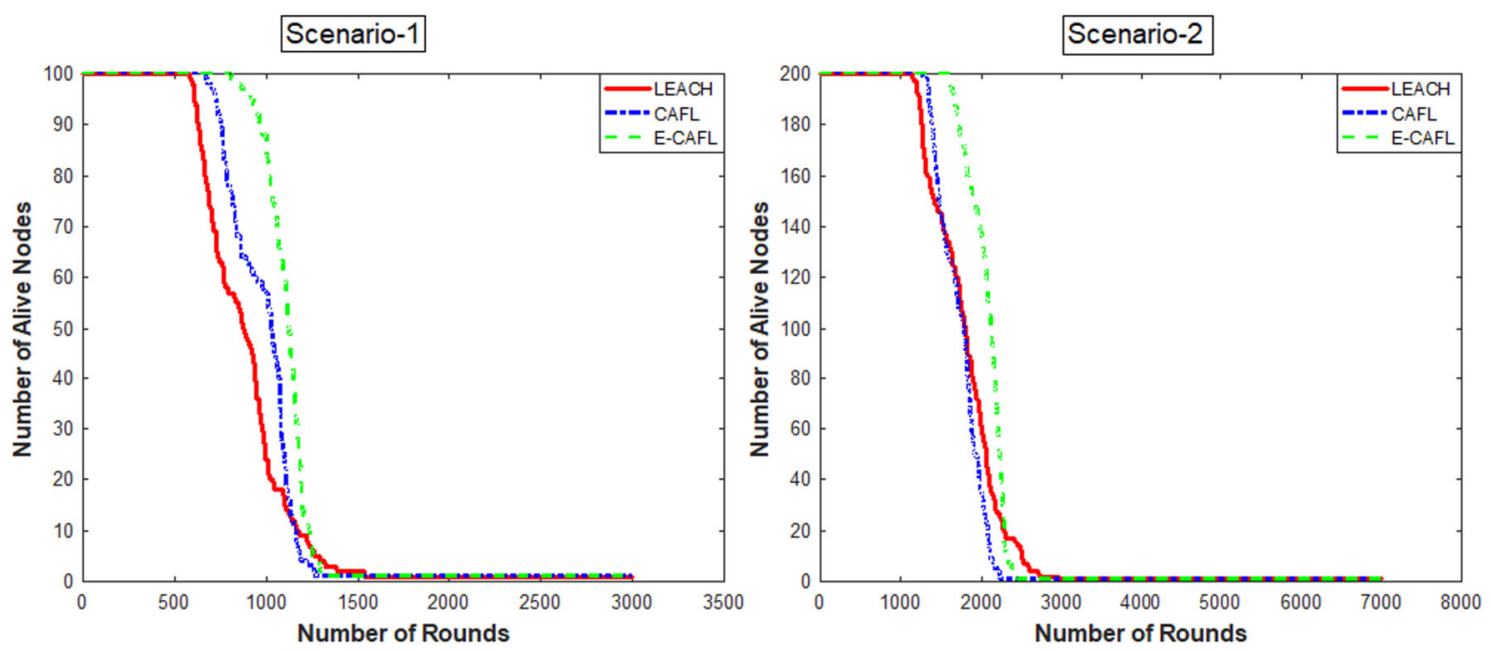

FIG. 5.1. Number of alive nodes

579 and 641 round respectively for scenario 1 whereas for scenario 2, stability period is achieved till 1502 rounds where as in case of CAFL and LEACH it is 1133 and 1220 rounds for death of first node. From these results we can conclude that E-CAFL dissipated energy in balanced manner. Both the protocols have performed poor due to non-consideration of node density which also plays an important role. With early expiration of SN, network becomes unstable as well as unreliable due to poor coverage. Better reliability and stability requires perishing of SN after later round.

5.2. Average Energy dissipation rate. Wireless communication fritters a large amount of energy. With the increase of rounds, average energy of the network decreases resulting in death of nodes. Figure 5.2 depicts the average energy dissipation rate of the network. When both the scenarios are considered, we can see that
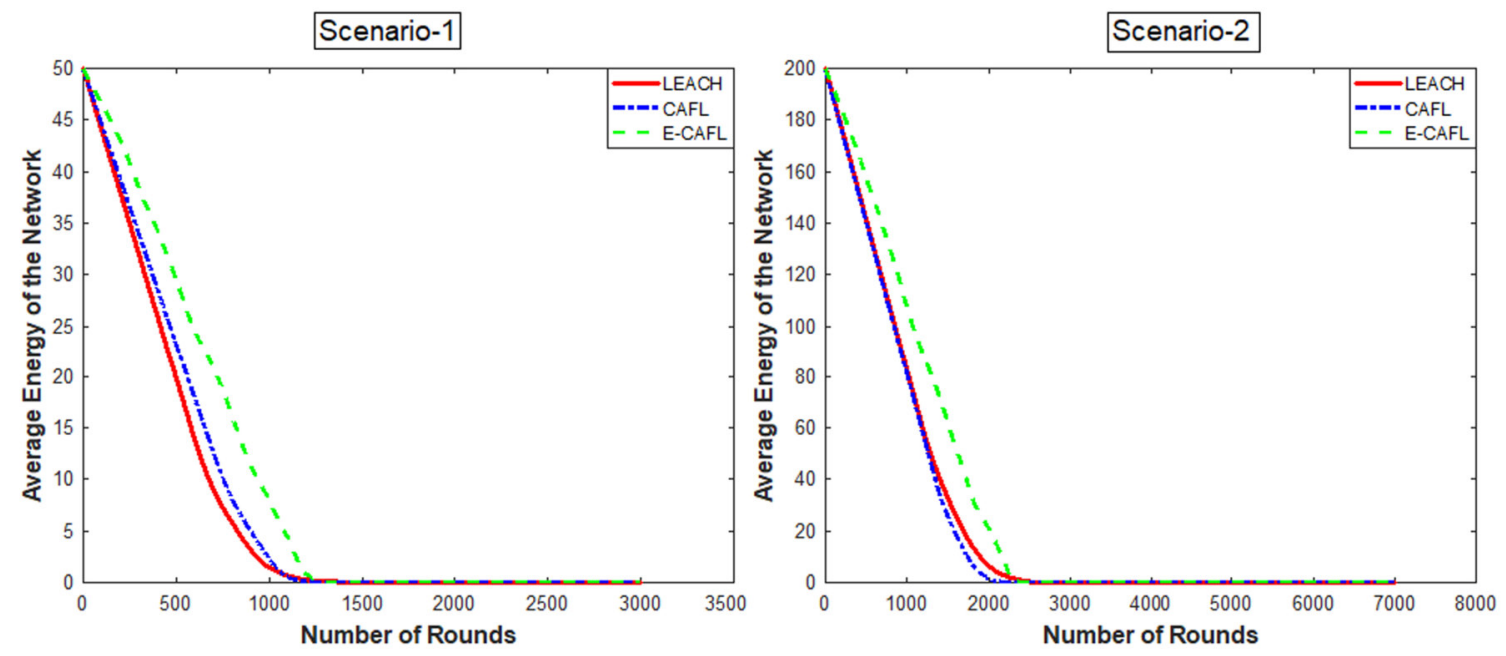

FIG. 5.2. Average energy dissipation rate 
E-CAFL dissipates less energy per round as compared to CAFL and LEACH protocol which reveals balanced load distribution of the proposed protocol resulting in longer stability period.

5.3. FND, QND and HND. After establishing the network, target is to gather more information from the field where network lifetime is essential. Premature expiration of SN may lead to uncovered regions of the field resulting in poor performance. Figure 5.3 shows the first node death, quarter node death and half node death metrics gathered from simulation experiments. In scenario 1, E-CAFL achieves 29.56\% and 21.04\%
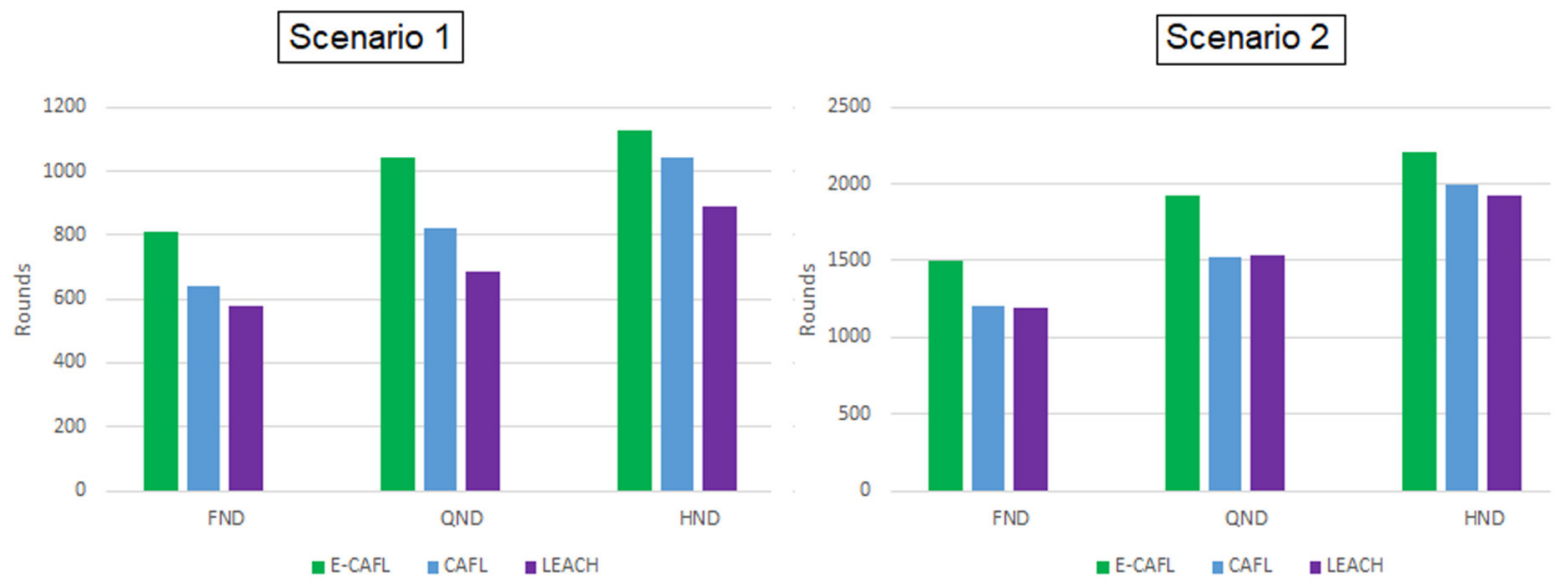

FIG. 5.3. FND, QND and $H N D$

improvement over LEACH and CAFL in FND where as it is $21.02 \%$ and $20.42 \%$ for scenario 2 . QND is prolonged by $34.06 \%$ and $21.20 \%$ as compared to LEACH and CAFL for scenario 1 , likewise for scenario 2 , it is $19.89 \%$ and $20.83 \%$ better than LEACH and CAFL respectively. E-CAFL protracts HND by $21.20 \%$ and $8.2 \%$ in comparison with LEACH and CAFL respectively for scenario 1. Likewise for scenario 2, it is improved by $13.12 \%$ and $10.02 \%$ over LEACH and CAFL respectively. These simulation results illuminate the proficiency of E-CAFL in better lifetime and protracted stable region.

5.4. Throughput of the network. More successful delivery of packet to BS demonstrates better throughput of the network. As we can see in Figure 5.4, for scenario 1, E-CAFL delivers $38.28 \%$ and $34.66 \%$ more packet as compared to LEACH and CAFL respectively which means large amount of information is collected at BS. Similarly, for scenario 2, E-CAFL has better throughput of $34.54 \%$ and $28.68 \%$ over LEACH and CAFL protocol respectively.

6. Conclusion. For proliferation of energy efficiency of the network and balanced energy consumption by the SN, fuzzy based clustering algorithm (E-CAFL) is propound. E-CAFL improves the CAFL protocol by considering the node density while making any decision be it rank while selecting $\mathrm{CH}$ candidature or chance while joining clusters by member nodes. Also, randomization in protocol may sometimes lead to zero $\mathrm{CH}$ which is eradicated in E-CAFL protocol. It is not possible always to place the BS within the network as there are some applications where WSN is unattended and SN are deployed on the fly. So in proposed work, BS is kept at distant place in order to satisfy all kind of applications. From the simulation experiments, the designed protocol (E-CAFL) performs better than its comparative i.e. LEACH and CAFL. E-CAFL significantly enhances the stability period in both the scenarios with better throughput by balancing the load of the network.

\section{REFERENCES}

[1] J. Yick, B. MukherJee, And D. Ghosal, Wireless sensor network survey, Computer networks, 52 (2008), pp. $2292-2330$.

[2] P. S. Mehra, M. N. DoJA, AND B. Alam, Zonal based approach for clustering in heterogeneous WSN, International Journal of Information Technology, (2017), pp. 1-9. 

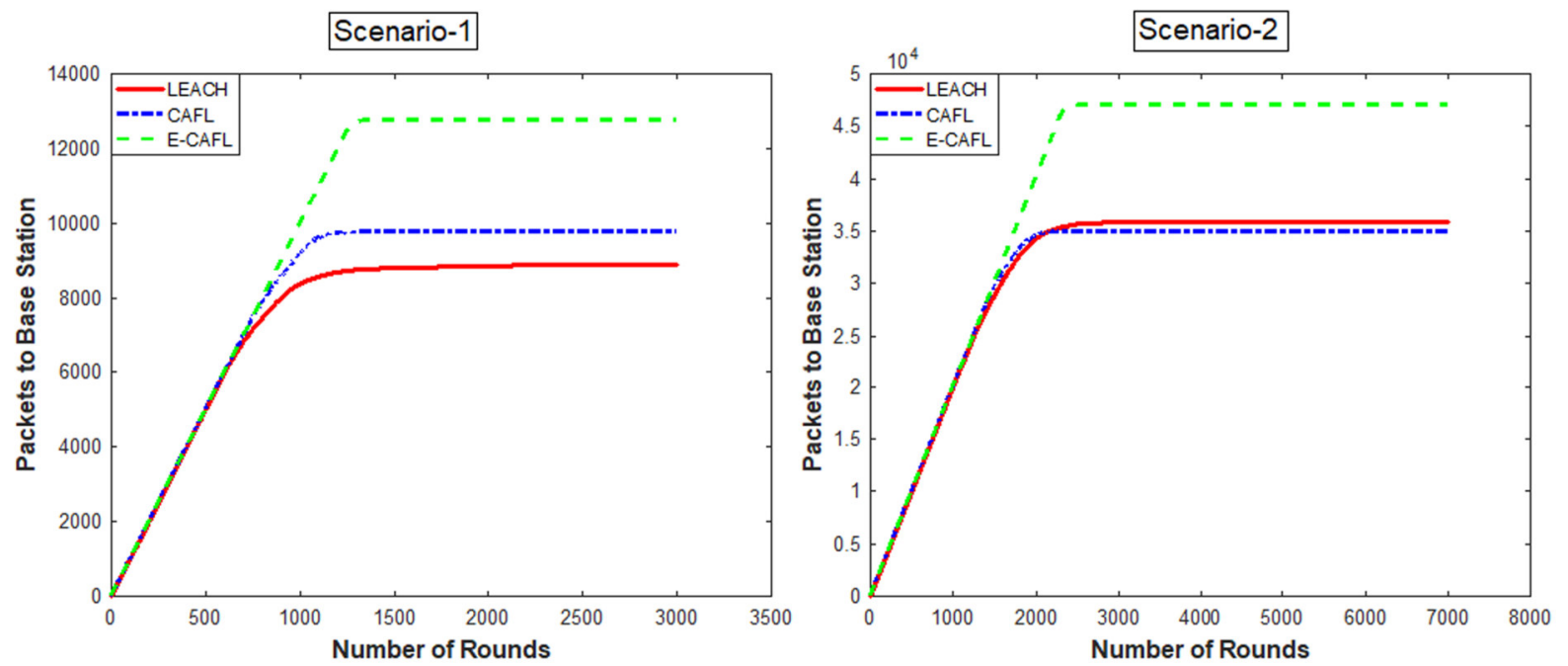

FIG. 5.4. Throughput of the network

[3] S. Tanwar, N. Kumar, And J. J. Rodrigues, A systematic review on heterogeneous routing protocols for wireless sensor network, Journal of Network and Computer Applications, 53 (2015), pp. 39-56.

[4] S. Arjunan and S. Pothula, A survey on unequal clustering protocols in Wireless Sensor Networks, Journal of King Saud University - Computer and Information Sciences, (2017).

[5] S. Tanwar, S. Tyagi, N. Kumar, and M. S. Obaidat, LA-MHR: Learning Automata Based Multilevel Heterogeneous Routing for Opportunistic Shared Spectrum Access to Enhance Lifetime of WSN, IEEE Systems Journal, (2018), pp. 1-11.

[6] P. S. Mehra, M. N. Doja, And B. Alam, Codeword Authenticated Key Exchange (CAKE) light weight secure routing protocol for WSN, International Journal of Communication Systems, 32 (2019).

[7] R. Want, K. Farkas, and C. Narayanaswami, Guest Editors' Introduction: Energy Harvesting and Conservation, IEEE Pervasive Computing, 4 (2005), pp. 14-17.

[8] M. Elshrkawey, S. M. Elsherif, and M. Elsayed Wahed, An Enhancement Approach for Reducing the Energy Consumption in Wireless Sensor Networks, Journal of King Saud University - Computer and Information Sciences, 30 (2018), pp. 259267.

[9] V. Raghunathan, C. Schurgers, Sung Park, and M. Srivastava, Energy-aware wireless microsensor networks, IEEE Signal Processing Magazine, 19 (2002), pp. 40-50.

[10] G. J. Pottie AND W. J. Kaiser, Wireless integrated network sensors, Communications of the ACM, 43 (2000), pp. 51-58.

[11] S. TANWAR, N. Kumar, AND J.-W. NiU, EEMHR: Energy-efficient multilevel heterogeneous routing protocol for wireless sensor networks, International Journal of Communication Systems, 27 (2014), pp. 1289-1318.

[12] P. S. Mehra, M. N. Doja, And B. Alam, Stable Period Enhancement for Zonal (SPEZ)-Based Clustering in Heterogeneous WSN, in Proceedings of First International Conference onSmart System, Innovations and Computing, (2018), pp. 887-896.

[13] H. El Alami And A. Najid, Fuzzy Logic Based Clustering Algorithm for Wireless Sensor Networks, International Journal of Fuzzy System Applications (IJFSA), 6 (2017), pp. 63-82.

[14] W. Heinzelman, A. Chandrakasan, and H. Balakrishnan, Energy-efficient communication protocol for wireless microsensor networks, in Proceedings of the 33rd Annual Hawaii International Conference on System Sciences, vol. vol.1, IEEE Comput. Soc, 2000, p. 10.

[15] I. Gupta, D. Riordan, and S. Sampalli, Cluster-Head Election Using Fuzzy Logic for Wireless Sensor Networks, in 3rd Annual Communication Networks and Services Research Conference (CNSR'05), IEEE, 2005, pp. 255-260.

[16] J. Kim, S. Park, Y. Han, and T. Chung, CHEF: Cluster Head Election mechanism using Fuzzy logic in Wireless Sensor Networks, in Proceedings of 10th International Conference on Advanced Communication Technology, 2008, pp. 654-659.

[17] G. Ran, H. Zhang, And S. Gong, Improving on LEACH Protocol of Wireless Sensor Networks Using Fuzzy Logic, Journal of Information \& Computational Science, (2010).

[18] J.-S. Lee And W.-L. Cheng, Fuzzy-Logic-Based Clustering Approach for Wireless Sensor Networks Using Energy Predication, IEEE Sensors Journal, 12 (2012), pp. 2891-2897.

[19] H. BAGCi And A. YAZICI, An energy aware fuzzy approach to unequal clustering in wireless sensor networks, Applied Soft Computing, 13 (2013), pp. 1741-1749.

[20] O. M. ALIA, A decentralized fuzzy c-means-based energy-efficient routing protocol for wireless sensor networks, The Scientific World Journal,(2014).

[21] S. A. Sert, H. BAGCI, And A. YazicI, Mofca: Multi-objective fuzzy clustering algorithm for wireless sensor networks, Applied Soft Computing, 30 (2015), pp. 151-165.

[22] S. Singh, S. Chand, And B. Kumar, Energy Efficient Clustering Protocol Using Fuzzy Logic for Heterogeneous WSNs, 
Wireless Personal Communications, 86 (2016), pp. 451-475.

[23] O. YOUNIS AND S. FAHMY, HEED: a hybrid, energy-efficient, distributed clustering approach for ad hoc sensor networks, Mobile Computing, IEEE Transactions on, 3 (2004), pp. 366-379.

[24] B. Baranidharan And B. SAnthi, DUCF: Distributed load balancing Unequal Clustering in wireless sensor networks using Fuzzy approach, Applied Soft Computing, 40 (2016), pp. 495-506.

[25] M. MiRzAiE AND S. M. MAZINANI, MCFL: an energy efficient multi-clustering algorithm using fuzzy logic in wireless sensor network, Wireless Networks, (2017), pp. 1-16.

[26] S. A. Sahayya Arul Mary and J. B. Gnanadurai, Enhanced Zone Stable Election Protocol based on Fuzzy Logic for Cluster Head Election in Wireless Sensor Networks, International Journal of Fuzzy Systems, 19 (2017), pp. 799-812.

[27] Y. K. TAmandani, M. U. Bokhari, And Q. M. Shallal, Two-step fuzzy logic system to achieve energy efficiency and prolonging the lifetime of WSNs, Wireless Networks, 23 (2017), pp. 1889-1899.

[28] P. S. Mehra, M. N. Doja, And B. Alam, Fuzzy based enhanced cluster head selection (FBECS) for WSN, Journal of King Saud University - Science, (2018).

[29] M. Mirzaie And S. M. MaZinani, MACHFL-FT: a fuzzy logic based energy-efficient protocol to cluster heterogeneous nodes in wireless sensor networks, Wireless Networks, (2018), pp. 1-13.

Edited by: Khaleel Ahmad

Received: Nov 15, 2018

Accepted: Feb 11, 2019 